\title{
The Batalin-Vilkovisky cohomology of the spinning particle
}

\section{Ezra Getzler \\ Department of Mathematics, Northwestern University, 2033 Sheridan Road, Evanston, Illinois, 60208 U.S.A. \\ E-mail: getzler@northwestern.edu}

ABSTRACT: We show that the axiom of Felder and Kazhdan [4] on the vanishing of the cohomology groups in negative degree associated to solutions of the classical master equation in the Batalin-Vilkovisky formalism is violated by the spinning particle in a flat background coupled to $D=1$ supergravity. In this model, there are nontrivial cohomology groups in all negative degrees, regardless of the dimension of the spacetime in which the spinning particle is propagating.

KEYwords: BRST Quantization, Supergravity Models, Differential and Algebraic Geometry

ARXIV EPRINT: 1511.02135 


\section{Contents}

1 The Batalin-Vilkovisky formalism 1

2 The classical master equation and the Batalin-Vilkovisky cohomology 2

3 The axiom of Felder and Kazhdan 4

4 Review of the spinning particle $\quad 5$

5 General covariance of the spinning particle $\quad 7$

6 The Batalin-Vilkovisky cohomology of the spinning particle: $d=0 \quad 8$

7 The Batalin-Vilkovisky cohomology of the spinning particle: $d>0 \quad 10$

In the 1970s, the BRST formalism was introduced as a tool in the pertubative quantization of gauge theories: it permits the use of rather general, non-gauge invariant regularizations, while still guaranteeing the gauge invariance of the pertubatively quantized amplitudes. The Batalin-Vilkovisky (BV) formalism was introduced in the 1980s to extend the BRST formalism to more general situations such as supersymmetric theories, especially those with extended supersymmetry, and supergravity.

The BV formalism has both classical and quantum versions. In this paper, we restrict attention to the classical BV formalism.

We will show that in a toy model of supergravity, namely the spinning particle, in which the worldsheet has dimension 1 , there is a series of cohomology classes in negative degrees, violating a basic axiom for the BV formalism which has been formulated recently by Felder and Kazhdan [4]. The nontrivial cohomology classes which we find are directly related to the possibility of formally inverting the superghost of the theory, associated to local supersymmetry.

This calculation seems to indicate that the BV prescription for associating ghost sectors to symmetries of the theory requires modification in the presence of local supersymmetry.

\section{The Batalin-Vilkovisky formalism}

The basic characteristic of the BV formalism is the doubling of the number of fields of the theory. Whereas in the BRST formalism, one has a series of fields $\Phi_{i}$ with ghost number $\operatorname{gh}\left(\Phi_{i}\right) \geq 0$, in the BV formalism, these are supplemented by antifields $\Phi_{i}^{+}$, with ghost number $\operatorname{gh}\left(\Phi_{i}^{+}\right)<0$, in such a way that $\operatorname{gh}\left(\Phi_{i}\right)+\operatorname{gh}\left(\Phi_{i}^{+}\right)=-1$.

Fields with ghost number 0 are interpreted as physical fields, while fields with ghost number greater than 0 are interpreted as ghosts. (Ghosts properly speaking are fields of 
ghost number 1: fields of ghost number greater than 1 are higher ghosts.) There is a similar division between those antifields with ghost number -1 , corresponding to physical fields, and those with ghost number less than -1 , corresponding to ghosts.

Besides its ghost number, each field $\Phi_{i}$ carries a parity $\mathrm{p}\left(\Phi_{i}\right) \in\{0,1\}$, defined modulo 2, which distinguishes between bosonic and fermionic fields. The parity of the antifield $\Phi_{i}^{+}$ paired to a field $\Phi_{i}$ satisfies $\mathrm{p}\left(\Phi_{i}\right)=1-\mathrm{p}\left(\Phi_{i}^{+}\right)$.

In this paper, we focus on the classical BV formalism with a single independent variable $t$ (classical mechanics). Let $\partial$ denote the total derivative with respect to $t$. Denote by $\mathcal{A}^{j}$ the superspace of all differential expressions in the fields and antifields with $\operatorname{gh}(S)=j$. For example, if there is a single physical field $\phi$, then $\mathcal{A}^{j}$ consists of all expressions in the field $\phi$ and its derivatives $\left\{\partial^{\ell} \phi\right\}_{\ell>0}$, and in the antifield $\phi^{+}$and its derivatives $\left\{\partial^{\ell} \phi^{+}\right\}_{\ell>0}$, polynomial in $\left\{\partial^{\ell} \phi\right\}_{\ell>0} \cup\left\{\partial^{\ell} \phi^{+}\right\}_{\ell \geq 0}$, and homogenous of degree $-j$ in $\left\{\partial^{\ell} \phi^{+}\right\}_{\ell \geq 0}$. The sum $\mathcal{A}$ of the superspaces $\mathcal{A}^{j}$ for $j \in \mathbb{Z}$ is a graded commutative superalgebra.

The superspace $\mathcal{F}$ of functionals is the graded quotient $\mathcal{A} / \partial \mathcal{A}$ of the algebra $\mathcal{A}$ of currents by the subspace $\partial \mathcal{A}$ of total derivatives. Denote the image of $f \in \mathcal{A}$ in $\mathcal{F}$ by $\int f d t$.

The fields and antifields are canonical coordinates for the Batalin-Vilkovisky antibracket, which is a Poisson bracket of degree 1:

$$
\left\{\Phi_{i}, \Phi_{j}^{+}\right\}=-\left\{\Phi_{j}^{+}, \Phi_{i}\right\}=\delta_{i j}
$$

In the finite dimensional setting, the antibracket satisfies the following equations:

(skew symmetry)

(Jacobi)

(Leibniz)

$$
\begin{aligned}
\{f, g\} & =-(-1)^{(\mathrm{p}(f)+1)(\mathrm{p}(g)+1)}\{g, f\} \\
\{f,\{g, h\}\} & =\{\{f, g\}, h\}+(-1)^{(\mathrm{p}(f)+1)(\mathrm{p}(g)+1)}\{g,\{f, h\}\} \\
\{f, g h\} & =\{f, g\} h+(-1)^{(\mathrm{p}(f)+1) \mathrm{p}(g)} g\{f, h\} .
\end{aligned}
$$

In our setting, the space of functionals $\mathcal{F}$ no longer carries a product, and we must we give up the Leibniz formula; in particular, the antibracket is not characterized by the canonical relations alone. Neverthless, the following antibracket makes the space of functionals $\mathcal{F}$ into a graded Lie algebra:

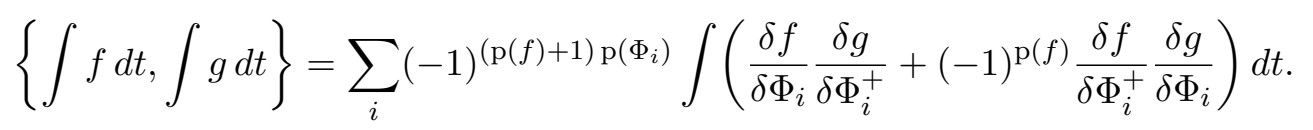

Here, $\delta / \delta \Phi_{i}$ and $\delta / \delta \Phi_{i}^{+}$are the variational derivatives, and the sum is over the fields of the theory.

\section{The classical master equation and the Batalin-Vilkovisky cohomology}

The Batalin-Vilkovisky formalism for classical theory involves the selection of a solution of the classical master equation

$$
\left\{\int S d t, \int S d t\right\}=0
$$


where $S \in \mathcal{A}^{0,0}$ is a differential expression in the fields and antifields with $\operatorname{gh}(S)=0$ and $\mathrm{p}(S)=0$. In other words, there is an expression $\tilde{S} \in \mathcal{A}^{1,1}$ such that

$$
\sum_{i}(-1)^{\mathrm{p}\left(\Phi_{i}\right)} \frac{\delta S}{\delta \Phi_{i}} \frac{\delta S}{\delta \Phi_{i}^{+}}=\partial \tilde{S}
$$

We may decompose $S$ into its components

$$
S=S_{[0]}+S_{[1]}+\ldots,
$$

where $S_{[k]}$ is homogenous of degree $k$ in the ghosts. The BRST formalism in its original form is the special case in which all ghost fields have ghost number 1, and $S_{[k]}=0$ for $k>2$.

Define an operator s: $\mathcal{F}^{k} \rightarrow \mathcal{F}^{k+1}$ by the formula

$$
\mathrm{s}=\left\{\int S d t,-\right\}
$$

By the classical master equation, this operator satisfies the equation $s^{2}=0$, and we may consider its cohomology groups $H^{*}(\mathcal{F}, \mathrm{s})$. These are called the BV cohomology associated to the solution $S$ of the classical master equation. The graded vector space $H^{*}(\mathcal{F}, \mathrm{s})$ is a graded Lie algebra, with bracket $\{-,-\}$ of degree 1 .

The operator s lifts to an evolutionary vector field on $\mathcal{A}$ of degree 1 , characterized by the formulas

$$
\mathrm{s} \Phi_{i}=(-1)^{\mathrm{p}\left(\Phi_{i}\right)+1} \frac{\delta S}{\delta \Phi_{i}^{+}} \quad \mathrm{s} \Phi_{i}^{+}=(-1)^{\mathrm{p}\left(\Phi_{i}\right)} \frac{\delta S}{\delta \Phi_{i}} .
$$

This vector field is cohomological:

$$
\mathrm{s}^{2}=0
$$

Since (2.1) holds, we may calculate the BV cohomology groups $H^{*}(\mathcal{F}, \mathrm{s})$ using the complex

$$
\mathcal{V}^{k}=\mathcal{A}^{k} \oplus \tilde{\mathcal{A}}^{k+1} \varepsilon,
$$

where

$$
\tilde{\mathcal{A}}^{k}= \begin{cases}\mathcal{A}^{0} / \mathrm{C}, & k=0, \\ \mathcal{A}^{k}, & k \neq 0 .\end{cases}
$$

The differential on this complex is given by the formula

$$
f+g \varepsilon \mapsto\left(\mathrm{s} f+(-1)^{\mathrm{p}(g)} \partial g\right)+\mathrm{s} g \varepsilon .
$$

In this way, we obtain a long exact sequence

$$
\begin{gathered}
\cdots \longrightarrow H^{-1}(\mathcal{A}, \mathrm{s}) \stackrel{\partial}{\longrightarrow} H^{-1}(\mathcal{A}, \mathrm{s}) \longrightarrow H^{-1}(\mathcal{F}, \mathrm{s}) \longrightarrow \\
H^{0}(\mathcal{A} / \mathrm{C}, \mathrm{s}) \stackrel{\partial}{\longrightarrow} H^{0}(\mathcal{A}, \mathrm{s}) \longrightarrow H^{0}(\mathcal{F}, \mathrm{s}) \longrightarrow \\
H^{1}(\mathcal{A}, \mathrm{s}) \stackrel{\partial}{\longrightarrow} H^{1}(\mathcal{A}, \mathrm{s}) \longrightarrow H^{1}(\mathcal{F}, \mathrm{s}) \longrightarrow \cdots
\end{gathered}
$$




\section{The axiom of Felder and Kazhdan}

The above formalism is extremely general. In an attempt to constrain the possible models to a class exhibiting the features of the classical field theories which are of interest in theoretical physics, Felder and Kazhdan [4] distilled from the work of Batalin and Vilkovisky an axiom for solutions of the classical master equation. Following their paper, we start by formulating the axiom in the finite-dimensional setting, in which fields are replaced by coordinates in a finite-dimensional graded supermanifold. (They restrict attention to graded manifolds, but for theories incorporating fermions, one must consider graded supermanifolds.)

Let $\mathcal{I} \subset \mathcal{A}$ be the ideal of $\mathcal{A}$ generated by the coordinates with positive ghost number, and let $\mathcal{A} / \mathcal{I}$ be the quotient graded superalgebra. The ideal $\mathcal{I}$ is closed under the action of $\mathrm{s}$, so the differential descends to the quotient graded superalgebra $\mathcal{A} / \mathcal{I}$, and we may define the cohomology $H^{*}(\mathcal{A} / \mathcal{I}, \mathrm{s})$, which is again a graded superalgebra. There is a quotient map

$$
H^{*}(\mathcal{A}, \mathrm{s}) \rightarrow H^{*}(\mathcal{A} / \mathcal{I}, \mathrm{s})
$$

which is in general neither surjective nor injective.

Following Batalin and Vilkovisky, Felder and Kazhdan propose the axiom that the cohomology of $\mathcal{A} / \mathcal{I}$ should vanish in negative degrees: if $k>0$,

$$
H^{-k}(\mathcal{A} / \mathcal{I}, \mathbf{s})=0
$$

The extension of this axiom to field theory is extremely powerful: for example, starting with the classical action for the Yang-Mills theory, it leads to the discovery of the gauge symmetry and its ghosts: the antifields of the ghosts are needed in order to kill the cohomology classes $\int\left(\epsilon, d_{A} A^{+}\right)$of degree -1 whose closure is a consequence of the second Bianchi identity for the curvature. (Here, $\epsilon$ is a section of the adjoint bundle $P \times_{G} \mathrm{~g}$ associated to the principal bundle $P$, and the antifield $A^{+}$is a three-form with values in $P \times_{G}$ g.)

By a spectral sequence argument, Felder and Kazdhan prove that their axiom implies the vanishing of the cohomology of $\mathcal{A}$ in negative degree: if $k>0$,

$$
H^{-k}(\mathcal{A}, \mathrm{s})=0
$$

Filtering $\mathcal{A}$ by powers of $\mathcal{I}$, one obtains a spectral sequence with $E_{2}$-term

$$
\bigoplus_{\ell=0}^{\infty} H^{*}\left(\mathcal{I}^{\ell} / \mathcal{I}^{\ell+1}, \mathrm{~s}\right) \Longrightarrow H^{*}(\mathcal{A}, \mathrm{s}) \text {. }
$$

Each of the complexes $\mathcal{I}^{\ell} / \mathcal{I}^{\ell+1}$ is a free module over $\mathcal{A} / \mathcal{I}$, with basis in strictly positive degrees: hence, if $H^{-k}(\mathcal{A} / \mathcal{I}, \mathbf{s})$ vanishes for all $k>0$, it follows that $H^{-k}(\mathcal{A}, \mathbf{s})$ vanishes for all $k>0$.

It is not hard to formulate the extension of the axiom of Felder and Kazhdan to the setting of classical field theory: here, we restrict attention to the case of a single independent variable $t$. Let $\mathcal{I} \subset \mathcal{A}$ be the ideal of $\mathcal{A}$ generated by the fields with positive ghost number 
and their partial derivatives, and let $\mathcal{A} / \mathcal{I}$ be the quotient graded superalgebra. There is a quotient complex

$$
\mathcal{F} \rightarrow \mathcal{A} /(\partial \mathcal{A}+\mathcal{I})
$$

and a quotient map

$$
H^{*}(\mathcal{F}, \mathrm{s}) \rightarrow H^{*}(\mathcal{A} /(\partial \mathcal{A}+\mathcal{I}), \mathrm{s})
$$

which is in general neither surjective nor injective.

The natural extension of the axiom of Felder and Kazhdan is that the cohomology of $\mathcal{A} /(\partial \mathcal{A}+\mathcal{I})$ should vanish in negative degrees: if $k>0$,

$$
H^{-k}(\mathcal{A} /(\partial \mathcal{A}+\mathcal{I}), \mathrm{s})=0 .
$$

The spectral sequence argument of Felder and Kazdhan extends to this setting, and shows that their axiom implies the vanishing of the cohomology of $\mathcal{F}$ in degree less than -1 : if $k>1$,

$$
H^{-k}(\mathcal{F}, \mathrm{s})=0
$$

Barnich et al. have shown that this holds for the pure Yang-Mills theory on $\mathbb{R}^{4}$, with arbitrary semisimple gauge group [1] (see also Costello [3, chapter 6, section 5]).

We will show that there exists a field theory in which the axiom of Felder and Kazhdan fails, in the sense that $H^{-k}(\mathcal{F}, \mathrm{s})$ is nonzero for all $k>1$.

\section{Review of the spinning particle}

We now investigate the axiom of Felder and Kazhdan in a toy model of supergravity, the spinning particle [2]. Consider the vector space $\mathbb{R}^{d}$ with non-degenerate inner product

$$
\left(e_{\mu}, e_{\nu}\right)=\eta_{\mu \nu}
$$

The spinning particle has physical fields $x^{\mu}=x^{\mu}(t)$ and $\theta^{\mu}=\theta^{\mu}(t)$ of parity 0 and 1 respectively, and action

$$
S=\frac{1}{2} \eta_{\mu \nu}\left(\partial x^{\mu} \partial x^{\nu}-\theta^{\mu} \partial \theta^{\nu}\right)
$$

where $\partial x^{\mu}$ and $\partial \theta^{\mu}$ are the derivatives of the fields $x^{\mu}$ and $\theta^{\mu}$ with respect to the independent variable $t$ parametrizing the time-line of the particle. We denote by $\mathcal{O}$ the algebra of functions in the variables $x^{\mu}$. There is a lot of flexibility in the choice of this algebra: we may take polynomials in the variables $x^{\mu}$, infinitely-differentiable functions, analytic functions, or even power series. All of our results will be independent of this choice. Thus, $\mathcal{A}$ is the graded polynomial algebra over $\mathcal{O}$ generated by the remaining variables of the theory, namely $\left\{\partial^{\ell} x^{\mu}\right\}_{\ell>0} \cup\left\{\partial^{\ell} \theta, \partial^{\ell} x_{\mu}^{+}, \partial^{\ell} \theta_{\mu}^{+}\right\}_{\ell \geq 0}$.

We prefer to work in a first-order formulation of this theory, which has an additional physical field $p_{\mu}$, with even parity, and the modified action

$$
S=p_{\mu} \partial x^{\mu}-\frac{1}{2} \eta_{\mu \nu} \theta^{\mu} \partial \theta^{\nu}-\frac{1}{2} \eta^{\mu \nu} p_{\mu} p_{\nu} .
$$


The differential $s$ on the fields and antifields of the theory is given by the formulas

$$
\begin{aligned}
& \mathrm{s} x_{\mu}^{+}=-\partial p_{\mu} \quad \mathrm{s} \theta_{\mu}^{+}=\eta_{\mu \nu} \partial \theta^{\nu} \quad \mathrm{s} p^{+\mu}=\partial x^{\mu}-\eta^{\mu \nu} p_{\nu} \\
& \mathbf{s} x^{\mu}=0 \quad \mathrm{~s} \theta^{\mu}=0 \quad \mathrm{~s} p_{\mu}=0 .
\end{aligned}
$$

This differential is an example of a Koszul complex, and the cohomology $H^{-k}(\mathcal{A} / \mathcal{I}, \mathrm{s})$ vanishes in negative degree, and in degree 0 is the graded polynomial ring in the functionals $\int x^{\mu} d t, \int \theta^{\mu} d t$ and $\int p_{\mu} d t$. In particular, the axiom of Felder and Kazhdan is seen to hold.

In order to have a theory with local reparametrization invariance and local supersymmetry, we may couple the spinning particle to supergravity. Of course, the gravitational field in worldsheet dimension 1 has no dynamical content: but we will see that the ghosts for the local supersymmetry of the theory considerably complicate matters.

The supergravity multiplet consists of a pair of physical fields $e$ and $\psi$, of parity $\mathrm{p}(e)=0$ and $\mathrm{p}(\psi)=1$. These fields, respectively a 1-form and a function, may be identified with the graviton and the gravitino of $D=1$ supergravity. The new action is

$$
S_{[0]}=p_{\mu} \partial x^{\mu}-\frac{1}{2} \eta_{\mu \nu} \theta^{\mu} \partial \theta^{\nu}-\frac{1}{2} e \eta^{\mu \nu} p_{\mu} p_{\nu}+\psi p_{\mu} \theta^{\mu} .
$$

The differential is now

$$
\begin{aligned}
& \mathrm{s}_{[0]} e^{+}=-\frac{1}{2} \eta^{\mu \nu} p_{\mu} p_{\nu} \quad \mathrm{s}_{[0]} \psi^{+}=-p_{\mu} \theta^{\mu} \\
& \mathrm{s}_{[0]} x_{\mu}^{+}=-\partial p_{\mu} \quad \mathbf{s}_{[0]} \theta_{\mu}^{+}=\eta_{\mu \nu} \partial \theta^{\nu}+\psi p_{\mu} \quad \mathbf{s}_{[0]} p^{+\mu}=\partial x^{\mu}-e \eta^{\mu \nu} p_{\nu}+\psi \theta^{\mu} .
\end{aligned}
$$

The variation $\mathrm{s}_{[0]} e^{+}$is familiar as the stress-energy tensor. The local gauge symmetries of this model correspond to cohomology classes of $s_{[0]}$ at ghost number -1 :

$$
\mathrm{s}_{[0]}\left(\partial e^{+}-\eta^{\mu \nu} x_{\mu}^{+} p_{\nu}\right)=0 \quad \mathbf{s}_{[0]}\left(\partial \psi^{+}+\eta^{\mu \nu} \theta_{\mu}^{+} p_{\nu}-x_{\mu}^{+} \theta^{\mu}+2 e^{+} \psi\right)=0 .
$$

These cohomology classes are killed by the introduction of ghosts $c$ and $\gamma$, with ghostnumber 1 and parity $\mathrm{p}(c)=1$ and $\mathrm{p}(\gamma)=0$, and the corresponding antifields, and the addition to the action of the term

$$
S_{[1]}=\left(\partial e^{+}-\eta^{\mu \nu} x_{\mu}^{+} p_{\nu}\right) c+\left(\partial \psi^{+}+\eta^{\mu \nu} \theta_{\mu}^{+} p_{\nu}-x_{\mu}^{+} \theta^{\mu}+2 e^{+} \psi\right) \gamma .
$$

This adds the following terms to the differential:

$$
\begin{aligned}
\mathrm{s}_{[1]} c^{+} & =\partial e^{+}-\eta^{\mu \nu} x_{\mu}^{+} p_{\nu} & \mathrm{s}_{[1]} \gamma^{+} & =\partial \psi^{+}+\eta^{\mu \nu} \theta_{\mu}^{+} p_{\nu}-x_{\mu}^{+} \theta^{\mu}+2 e^{+} \psi \\
\mathrm{s}_{[1]} \psi^{+} & =2 e^{+} \gamma & & \\
\mathrm{s}_{[1]} \theta_{\mu}^{+} & =-x_{\mu}^{+} \gamma & \mathrm{s}_{[1]} p^{+\mu} & =-\eta^{\mu \nu} x_{\nu}^{+} c+\eta^{\mu \nu} \theta_{\nu}^{+} \gamma \\
\mathrm{s}_{[1]} x^{\mu} & =-\eta^{\mu \nu} p_{\nu} c-\theta^{\mu} \gamma & \mathrm{s}_{[1]} \theta^{\mu} & =-\eta^{\mu \nu} p_{\nu} \gamma \\
\mathrm{s}_{[1]} & =-\partial c+2 \psi \gamma & \mathrm{s}_{[1]} \psi & =\partial \gamma .
\end{aligned}
$$

The variation $\mathrm{s}_{[1]} c^{+}$reflects the conservation of the stress-energy tensor. The definition of the action is completed by the addition of the term

$$
S_{[2]}=-c^{+} \gamma^{2}
$$


yielding a solution $S=S_{[0]}+S_{[1]}+S_{[2]}$ of the classical master equation

$$
\left\{\int S d t, \int S d t\right\}=0
$$

This adds the following terms to the differential, rendering it nilpotent:

$$
\mathrm{s}_{[2]} \gamma^{+}=-2 c^{+} \gamma \quad \mathrm{s}_{[2]} c=\gamma^{2} .
$$

In this model, $\mathcal{A}$ is the graded polynomial algebra over $\mathcal{O}$ generated by the remaining variables of the theory, namely

$$
\begin{aligned}
\left\{\partial^{\ell} x^{\mu}\right\}_{\ell>0} & \cup\left\{\partial^{\ell} \theta, \partial^{\ell} p_{\mu}, \partial^{\ell} e, \partial^{\ell} \psi, \partial^{\ell} c, \partial^{\ell} \gamma\right\}_{\ell \geq 0} \\
& \cup\left\{\partial^{\ell} x_{\mu}^{+}, \partial^{\ell} \theta_{\mu}^{+}, \partial^{\ell} p^{+\mu}, \partial^{\ell} e^{+}, \partial^{\ell} \psi^{+}, \partial^{\ell} c^{+}, \partial^{\ell} \gamma^{+}\right\}_{\ell \geq 0} .
\end{aligned}
$$

As it happens, the action $S$ satisfies not just the classical master equation but also the quantum master equation, since for each field $\Phi$ of the model, we have

$$
\frac{\partial^{2} S}{\partial \Phi \partial \Phi^{+}}=0
$$

This is characteristic of quantum mechanics, where one does not meet with anomalies in the course of quantization.

\section{General covariance of the spinning particle}

The Lie algebra of vector fields on the real line acts on the spinning particle by reparametrization of the independent variable $t$. Reflecting this, this Lie algebra acts on $\mathcal{A}$ by evolutionary vector fields: the vector field $-\xi \partial / \partial t$ on the real line acts by the evolutionary vector field

$$
\begin{aligned}
T(\xi) \Phi= & \xi\left(\partial x^{\mu} \frac{\partial}{\partial x^{\mu}}+\partial \theta^{\mu} \frac{\partial}{\partial \theta^{\mu}}+\partial p_{\mu} \frac{\partial}{\partial p_{\mu}}+\partial x_{\mu}^{+} \frac{\partial}{\partial x_{\mu}^{+}}+\partial \theta_{\mu}^{+} \frac{\partial}{\partial \theta_{\mu}^{+}}+\partial p^{+\mu} \frac{\partial}{\partial p^{+\mu}}\right. \\
& \left.+\partial e \frac{\partial}{\partial e}+\partial \psi \frac{\partial}{\partial \psi}+\partial e^{+} \frac{\partial}{\partial e^{+}}+\partial \psi^{+} \frac{\partial}{\partial \psi^{+}}+\partial c \frac{\partial}{\partial c}+\partial \gamma \frac{\partial}{\partial \gamma}+\partial c^{+} \frac{\partial}{\partial c^{+}}+\partial \gamma^{+} \frac{\partial}{\partial \gamma^{+}}\right) \\
& -\partial \xi\left(e \frac{\partial}{\partial e}+\psi \frac{\partial}{\partial \psi}+x_{\mu}^{+} \frac{\partial}{\partial x_{\mu}^{+}}+\theta_{\mu}^{+} \frac{\partial}{\partial \theta_{\mu}^{+}}+p^{+\mu} \frac{\partial}{\partial p^{+\mu}}+\partial c^{+} \frac{\partial}{\partial c^{+}}+\partial \gamma^{+} \frac{\partial}{\partial \gamma^{+}}\right) .
\end{aligned}
$$

(We may think of $\xi$ either as a function of the independent variable $t$, in which case $\partial \xi=\xi^{\prime}$, or, more in the spirit of this article, as an auxiliary bosonic field.) In particular, $T(1)=\partial$.

Consider the expression

$$
G=x_{\mu}^{+} p^{+\mu}-\frac{1}{2} \eta^{\mu \nu} \theta_{\mu}^{+} \theta_{\nu}^{+}+c^{+} e+\gamma^{+} \psi .
$$

The general covariance of the spinning particle is expressed through the formula

$$
\{\{S, \xi G\}, f\}=T(\xi) f
$$


which follows from the identity

$$
\{S, \xi G\}=-\xi\left(x_{\mu}^{+} \partial x^{\mu}+\theta_{\mu}^{+} \partial \theta^{\mu}+p^{+\mu} \partial p_{\mu}-\partial e^{+} e+c^{+} \partial c-\partial \psi^{+} \psi+\gamma^{+} \partial \gamma\right) .
$$

As a special case, we have

$$
\{\{S, G\}, f\}=\partial f .
$$

This corresponds to the commutator

$$
[\mathbf{s}, \mathrm{g}]=\partial
$$

among evolutionary vector fields, where $\mathrm{g}$ is the vector field $\mathrm{g}(f)=\{G, f\}$ associated to $G$ :

$$
\mathrm{g}=p^{+\mu} \frac{\partial}{\partial x^{\mu}}-x_{\mu}^{+} \frac{\partial}{\partial p_{\mu}}+\eta^{\mu \nu} \theta_{\mu}^{+} \frac{\partial}{\partial \theta^{\mu}}+c^{+} \frac{\partial}{\partial e^{+}}-e \frac{\partial}{\partial c}+\gamma^{+} \frac{\partial}{\partial \psi^{+}}+\psi \frac{\partial}{\partial \gamma} .
$$

We see that the total derivative $\partial$ acts trivially on the cohomology $H^{*}(\mathcal{A}, \mathrm{s})$.

\section{The Batalin-Vilkovisky cohomology of the spinning particle: $d=0$}

In this section, we calculate the BV cohomology of the spinning particle when the matter fields $x^{\mu}, \theta^{\mu}, p_{\mu}$ are absent, that is, when the dimension $d$ of the target space $\mathbb{R}^{d}$ is zero. We will see that the cohomology groups are nontrivial in all negative degrees.

Consider the following elements of the localization $\mathcal{A}_{\gamma}$ of the complex $\mathcal{A}$ obtained by inversion of $\gamma$ :

$$
\begin{array}{rlrl}
A_{k} & =\left(\psi^{+}\right)^{k+1} c \gamma^{-1} \in \mathcal{A}_{\gamma}^{-k-1}, & & k \geq-1, \\
B_{k} & =\frac{1}{k}\left(\psi^{+}\right)^{k} \gamma^{-1} \in \mathcal{A}_{\gamma}^{-k-1}, & k \geq 1 .
\end{array}
$$

The BV differentials of these expressions are in $\mathcal{A}$ itself:

$$
\begin{aligned}
\alpha_{k} & =2(k+1)\left(\psi^{+}\right)^{k} e^{+} c+\left(\psi^{+}\right)^{k+1} \gamma \in \mathcal{A}^{-k}, & & k \geq-1, \\
\beta_{k} & =\left(\psi^{+}\right)^{k-1} e^{+} \in \mathcal{A}^{-k}, & & k \geq 1 .
\end{aligned}
$$

It follows that $\alpha_{k}$ and $\beta_{k}$ are cocycles: it is not difficult to see that they are not coboundaries in $\mathcal{A}$ itself.

Theorem. The cohomology group $H^{-k}(\mathcal{A}, \mathrm{s})$ is spanned by the cocycles $\alpha_{k}$ and $\beta_{k}$, and the cocycle 1 in degree 0.

Proof. The differential s is a quadratic perturbation of a Koszul differential, and its cohomology may be calculated by a spectral sequence with $E_{0}$ equal to $\mathcal{A}$ with the differential $\mathrm{s}_{0}$ obtained by retaining only linear terms in the formula for $s$ :

$$
\mathrm{s}_{0} c^{+}=\partial e^{+} \quad \mathrm{s}_{0} \gamma^{+}=\partial \psi^{+} \quad \mathrm{s}_{0} e=-\partial c \quad \mathrm{~s}_{0} \psi=\partial \gamma .
$$

The cohomology $E_{1}$ of the differential $\mathrm{s}_{0}$ is a graded polynomial ring in generators $\mathrm{E}^{+}=$ $\int e^{+} d t$ and $\Psi^{+}=\int \psi^{+} d t$, in degree -1 , and $\mathbf{C}=\int c d t$ and $\Gamma=\int \gamma d t$, in degree 1 . 
The differential $\mathrm{s}_{1}$ on $E_{1}$ is given by the formulas

$$
\mathrm{s}_{1} \Psi^{+}=2 \mathrm{E}^{+} \Gamma \quad \mathrm{s}_{1} \mathrm{C}=\Gamma^{2} .
$$

A cochain $z$ of degree $-k$ for $k>0$ has the following general form:

$$
\begin{aligned}
z=u_{k}\left(\Psi^{+}\right)^{k}+v_{k}\left(\Psi^{+}\right)^{k-1} \mathrm{E}^{+} & \\
& +\sum_{j=1}^{\infty}\left(\left(\Psi^{+}\right)^{k+j}\left(u_{k+j} \Gamma^{j}+U_{k+j} \mathrm{C} \Gamma^{j-1}\right)+\left(\Psi^{+}\right)^{k+j-1} \mathrm{E}^{+}\left(v_{k+j} \Gamma^{j}+V_{k+j} \mathrm{C} \Gamma^{j-1}\right)\right) .
\end{aligned}
$$

Setting $\mathbf{s}_{1} z=0$, we see that $U_{k+j}=V_{k+j}-2(k+j) u_{k+j}=0$, and that

$$
z=u_{k+1} \alpha_{k}+v_{k} \beta_{k}+s_{1}\left(\sum_{j=2}^{\infty} u_{k+j}\left(\Psi^{+}\right)^{k+j} \mathrm{C} \Gamma^{j-2}+\sum_{j=1}^{\infty} \frac{v_{k+j}}{2(k+j)}\left(\Psi^{+}\right)^{k+j} \Gamma^{j-1}\right) .
$$

This shows that if $k>0, H^{-k}(\mathcal{A}, \mathrm{s})$ is spanned by $\alpha_{k}$ and $\beta_{k}$.

A cochain $z$ of degree 0 has the following general form:

$$
z=u_{0}+\sum_{j=1}^{\infty}\left(\left(\Psi^{+}\right)^{j}\left(u_{j} \Gamma^{j}+U_{j} \mathrm{C} \Gamma^{j-1}\right)+\left(\Psi^{+}\right)^{j-1} \mathrm{E}^{+}\left(v_{j} \Gamma^{j}+V_{j} \mathrm{C} \Gamma^{j-1}\right)\right) .
$$

Setting $\mathrm{s}_{1} z=0$, we see that

$$
z=u_{0}+u_{1} \alpha_{0}+s_{1}\left(-\sum_{j=2}^{\infty} u_{j}\left(\Psi^{+}\right)^{k} \mathrm{C} \Gamma^{j-2}+\sum_{j=1}^{\infty} \frac{v_{j}}{2 k}\left(\Psi^{+}\right)^{k} \Gamma^{j-1}\right) .
$$

This shows that $H^{0}(\mathcal{A}, \mathrm{s})$ is spanned by 1 and $\alpha_{0}$. Similar calculations show that $H^{1}(\mathcal{A}, \mathrm{s})$ is spanned by $\alpha_{-1}$, and that $H^{\ell}(\mathcal{A}, \mathrm{s})$ vanishes for $\ell>1$.

Let $\mathrm{g}$ be the vector field (5.2), which in the present setting equals

$$
\mathrm{g}=c^{+} \frac{\partial}{\partial e^{+}}-e \frac{\partial}{\partial c}+\gamma^{+} \frac{\partial}{\partial \psi^{+}}+\psi \frac{\partial}{\partial \gamma}
$$

and consider the transgressions of the cocycles $\alpha_{k}$ and $\beta_{k}$ :

$$
\begin{aligned}
\tilde{\alpha}_{k}=\mathrm{g}\left(\alpha_{k-1}\right) \in \mathcal{A}^{-k}, & k \geq 0, \\
\tilde{\beta}_{k}=\mathrm{g}\left(\beta_{k-1}\right) \in \mathcal{A}^{-k}, & k \geq 2 .
\end{aligned}
$$

Corollary. The cohomology group $H^{-k}(\mathcal{F}, \mathbf{s})$ is spanned by the cocycles $\int \alpha_{k} d t, \int \beta_{k} d t$, $\int \tilde{\alpha}_{k} d t$ and $\int \tilde{\beta}_{k} d t$, and the cocycle $\int 1 d t$ in degree 0 .

Proof. We consider the long exact sequence (2.2). By (5.1), the morphisms

$$
\partial: H^{-k}(\mathcal{A}, \mathrm{s}) \rightarrow H^{-k}(\mathcal{A}, \mathrm{s})
$$

vanish. This implies that the classes $\int \alpha_{k} d t$ and $\int \beta_{k} d t$ and their transgressions $\int \tilde{\alpha}_{k} d t$ and $\int \tilde{\beta}_{k} d t$ span $H^{-k}(\mathcal{F}, \mathrm{s})$, together with $\int 1 d t$ in degree 0 . 
It turns out that the graded Lie bracket on $H^{*}(\mathcal{F}, \mathrm{s})$ is nontrivial.

Theorem. We have the following nonzero graded Lie brackets in $H^{*}(\mathcal{F}, \mathrm{s})$ :

$$
\begin{array}{ll}
\left\{\tilde{\alpha}_{k}, \alpha_{\ell}\right\}=(\ell-k) \alpha_{k+\ell} & \left\{\tilde{\alpha}_{k}, \tilde{\alpha}_{\ell}\right\}=(\ell-k) \tilde{\alpha}_{k+\ell} \\
\left\{\tilde{\alpha}_{k}, \beta_{\ell}\right\}=(2 k+\ell+1) \beta_{k+\ell} & \left\{\tilde{\alpha}_{k}, \tilde{\beta}_{\ell}\right\}=(2 k+\ell+1) \tilde{\beta}_{k+\ell}
\end{array}
$$

All other brackets are zero.

Proof. By (5.1), we see that

$$
\left\{\tilde{\alpha}_{k}, \alpha_{\ell}\right\}=-\mathrm{s}\left\{\mathrm{g}\left(A_{k}\right), \alpha_{\ell}\right\}+\partial\left\{A_{k}, \alpha_{\ell}\right\}=-\mathrm{s}\left\{\mathrm{g}\left(A_{k}\right), \alpha_{\ell}\right\},
$$

since $\left\{A_{k}, \alpha_{\ell}\right\}=0$. We have

$$
\begin{aligned}
\left\{\mathrm{g}\left(A_{k}\right), \alpha_{\ell}\right\} & =\left\{(k+1)\left(\psi^{+}\right)^{k} \gamma^{+} c \gamma^{-1}-\left(\psi^{+}\right)^{k+1} e \gamma^{-1}+\left(\psi^{+}\right)^{k+1} c \psi \gamma^{-2}, \alpha_{\ell}\right\} \\
& =(k+1) A_{k+\ell+1}-2(\ell+1) A_{k+\ell+1}+(\ell+1) A_{k+\ell+1} \\
& =(k-\ell) A_{k+\ell}
\end{aligned}
$$

and the formula for $\left\{\tilde{\alpha}_{k}, \alpha_{\ell}\right\}$ follows. Applying the vector field $\mathrm{g}$ to both sides of the formula for $\left\{\tilde{\alpha}_{k}, \alpha_{\ell}\right\}$, we obtain the formula for $\left\{\tilde{\alpha}_{k}, \tilde{\alpha}_{\ell}\right\}$.

Similarly, $\left\{\tilde{\alpha}_{k}, \beta_{\ell}\right\}=-\mathrm{s}\left\{\mathrm{g} A_{k}, \beta_{\ell}\right\}$. Since

$$
\begin{aligned}
\left\{\mathrm{g}\left(A_{k}\right), \beta_{\ell}\right\} & =\left\{(k+1)\left(\psi^{+}\right)^{k} \gamma^{+} c \gamma^{-1}-\left(\psi^{+}\right)^{k+1} e \gamma^{-1}+\left(\psi^{+}\right)^{k+1} c \psi \gamma^{-2}, \beta_{\ell}\right\} \\
& =-\left(\psi^{+}\right)^{k+\ell} \gamma^{-1}-(\ell-1)\left(\psi^{+}\right)^{k+\ell-1} e^{+} c \gamma^{-2}
\end{aligned}
$$

the formula for $\left\{\tilde{\alpha}_{k}, \beta_{\ell}\right\}$ follows. Finally, applying the vector field $\mathrm{g}$ to both sides of the formula for $\left\{\tilde{\alpha}_{k}, \beta_{\ell}\right\}$, we obtain the formula for $\left\{\tilde{\alpha}_{k}, \tilde{\beta}_{\ell}\right\}$. It is easily seen from the explicit formulas that all of the remaining brackets vanish.

\section{The Batalin-Vilkovisky cohomology of the spinning particle: $d>0$}

The cohomology classes $\alpha_{k}$ and $\beta_{k}$ which we constructed in the previous section have generalizations for the spinning particle in a positive-dimensional target, where $d>0$. Here, we discuss the case of a flat target: we generalize our results to targets with nontrivial (pseudo-)Riemannian metric, and background magnetic field, in a forthcoming paper.

Although the formulas for the cohomology classes of negative degree are quite complicated, they all involve an analogue of the volume form for $\mathbb{R}^{d}$ constructed from the field $\theta^{\mu}$ :

$$
\Omega=\theta^{1} \ldots \theta^{d} .
$$

Since $(\Omega)^{2}=0$ when $d>0$, it turns out that the Lie bracket on the cohomology $H^{*}(\mathcal{F}, \mathrm{s})$ is simpler in the general case.

Let $\iota_{\mu}=\partial / \partial \theta^{\mu}$. If $v$ is a vector with components $v_{\mu}$, define

$$
\iota(v)=\eta^{\mu \nu} v_{\mu} \iota_{\nu}
$$


In particular, $[\mathbf{s}, \iota(v)]=\iota(\mathbf{s})$. Note that $\mathbf{s} \Omega=\iota(p) \Omega \gamma$. If $f \in \mathcal{O}$, denote by $\operatorname{grad} f$ the vector with components $\partial f / \partial x^{\mu}$.

Given a function $f$ of the coordinates $x^{\mu}$ and $k \geq 0$, consider the following elements of $\mathcal{A}_{\gamma}^{-k-1}$ :

$$
\begin{aligned}
& A_{k}(f)=\left(\psi^{+}\right)^{k+1} c f \Omega \gamma^{-1}, \\
& Z_{k}(f)=(k+1)\left(\psi^{+}\right)^{k} f \Omega \gamma^{-1}+\left(\psi^{+}\right)^{k+1} c \iota(\operatorname{grad} f) \Omega \gamma^{-1} .
\end{aligned}
$$

The BV differentials of these expressions are in $\mathcal{A}^{-k}$ :

$$
\alpha_{k}(f)=\mathrm{s}\left(A_{k}(f)\right), \quad \zeta_{k}(f)=\mathrm{s}\left(Z_{k}(f)\right) .
$$

It follows that they are are cocycles in $\mathcal{A}$ : we will see that they represent nontrivial cohomology classes.

There are also cocycles in degrees 0 and 1 which have no analogue in the case where $d=0$. Let $\mathcal{R}$ be the quotient of the differential graded superalgebra $\mathcal{A}$ by the differential ideal generated by the fields

$$
\{e, \psi, c\} \cup\left\{x_{\mu}^{+}, \theta_{\mu}^{+}, p^{+\mu}, e^{+}, \chi^{+}, c^{+}, \gamma^{+}\right\} .
$$

Denote by $\mathrm{P}_{\mu}, \Theta^{\mu}, \mathrm{X}^{\mu}$ and $\Gamma$ the zero-modes $\int p_{\mu} d t, \int \theta^{\mu} d t, \int x^{\mu} d t$ and $\int \gamma d t$ respectively. Then $\mathcal{R}$ is the graded superalgebra

$$
\mathcal{O}\left[\Theta^{\mu}, \mathrm{P}_{\mu}, \Gamma\right] /\left(\mathrm{P}_{\mu} \Theta^{\mu}, \eta^{\mu \nu} \mathrm{P}_{\mu} \mathrm{P}_{\nu}, \Gamma^{2}\right)
$$

with differential $\mathrm{Q} \Gamma$, where $\mathrm{Q}$ is the differential operator

$$
\mathrm{Q}=\eta^{\mu \nu} \mathrm{P}_{\mu} \frac{\partial}{\partial \Theta^{\nu}}+\Theta^{\mu} \frac{\partial}{\partial \mathbf{X}^{\mu}}
$$

We may think of $\mathcal{R}$ as the ring of functions whose spectrum is a supersymmetric analogue of the light-cone. We will denote $\Theta^{1} \ldots \Theta^{d} \in \mathcal{R}^{0}$ by the same letter $\Omega$ as in $\mathcal{A}^{0}$.

There is an embedding $\xi$ of $H^{*}(\mathcal{R})$ into $H^{*}(\mathcal{A}, \mathrm{s})$, defined by mapping a function $u$ of the variables $\left\{\mathrm{X}^{\mu}, \Theta^{\mu}, \mathrm{P}_{\mu}\right\}$ to the corresponding function $\xi^{0}(u)$ of the variables $\left\{x^{\mu}, \theta^{\mu}, p_{\mu}\right\}$ in $\mathcal{A}$. If $\mathrm{Q} u=0$, it follows that $\xi^{0}(u)$ is a cocycle.

Since $\left(P_{\mu} \Theta^{\mu}\right) \xi^{0}(u)=-\mathrm{s}\left(\psi^{+} \xi^{0}(u)\right)$ and $\left(\eta^{\mu \nu} P_{\mu} P_{\nu}\right) \xi^{0}(u)=-2 \mathrm{~s}\left(e^{+} \xi^{0}(u)\right)$ are coboundaries, we see that $\xi^{0}$ induces a map from $H^{0}(\mathcal{R})$ to $H^{0}(\mathcal{A}, \mathrm{s})$. Note that $\xi^{0}(\iota(P) \Omega)=-\zeta_{0}(1)$.

We may also map a function $v$ of the variables $\left\{\mathrm{X}^{\mu}, \Theta^{\mu}, \mathrm{P}_{\mu}\right\}$ to the 1-cocycle

$$
\xi^{1}(v)=\gamma v+c \mathbf{Q} v
$$

By the formulas

$$
\xi^{1}(\mathrm{Q} v)=-\mathrm{s}\left(\xi^{1}(v)\right)
$$

and

$$
\xi^{1}\left(\mathrm{P}_{\mu} \Theta^{\mu} v\right)=\mathrm{s}\left(2 e^{+} c \xi^{0}(v)-\psi^{+} \xi^{1}(v)\right) \quad \xi^{1}\left(\eta^{\mu \nu} \mathrm{P}_{\mu} \mathrm{P}_{\nu} v\right)=-2 s\left(e^{+} \xi^{1}(v)\right),
$$

we see that $\xi^{1}$ induces a map from $H^{1}(\mathcal{R})$ to $H^{1}(\mathcal{A}, \mathrm{s})$. 


\section{Theorem.}

$$
H^{-k}(\mathcal{A}, \mathbf{s})= \begin{cases}\left\{\int\left(\alpha_{k}(f)+\zeta_{k}(g)\right) d t \mid f, g \in \mathcal{O}\right\} & k>0, \\ \left\{\int\left(\xi^{0}(u)+\alpha_{0}(f)+\zeta_{0}(g)\right) d t \mid u \in H^{0}(\mathcal{R}), f \in \mathcal{O}, g \in \mathcal{O} / \mathbb{C}\right\} & k=0, \\ \left\{\int \xi^{1}(v) d t \mid v \in H^{1}(\mathcal{R})\right\} & k=-1, \\ 0 & k<-1 .\end{cases}
$$

Proof. The proof uses a filtration on $\mathcal{A}$, defined by assigning to the fields the following filtration degrees:

\begin{tabular}{|c|c|c|}
\hline$\Phi$ & $\operatorname{deg}(\Phi)$ & $\operatorname{deg}\left(\Phi^{+}\right)$ \\
\hline$x$ & 0 & $2 \sigma$ \\
$\theta$ & $\sigma$ & $\sigma$ \\
$p$ & $2 \sigma$ & 0 \\
$e$ & $2-2 \sigma$ & $4 \sigma-1$ \\
$\psi$ & $2-\sigma$ & $3 \sigma-1$ \\
$c$ & $2-2 \sigma$ & $4 \sigma-1$ \\
$\gamma$ & $2-\sigma$ & $3 \sigma-1$ \\
\hline
\end{tabular}

We will see that the spectral sequence converges if $\sigma$ equals 1 or 0 . In the former case, the filtration degree is positive, while in the second case, the complementary degree is negative. The choice $\sigma=0$ is convenient in a sequel to this paper, where we discuss the case of a non-negative magnetic field.

The filtration degrees of the terms of the BV differential s are independent of $\sigma$, and lie between 0 and 2 . The differential $s_{0}$ on $E_{0}$ is the following Koszul differential:

$$
\begin{aligned}
& \mathrm{s}_{0} c^{+}=\partial e^{+} \quad \mathrm{s}_{0} \gamma^{+}=\partial \psi^{+} \\
& \mathrm{s}_{0} x_{\mu}^{+}=-\partial p_{\mu} \quad \mathrm{s}_{0} \theta_{\mu}^{+}=\eta_{\mu \nu} \partial \theta^{\nu} \quad \mathrm{s}_{0} p^{+\mu}=\partial x^{\mu} \quad \mathrm{s}_{0} e^{+}=0 \quad \mathrm{~s}_{0} \psi^{+}=0 \\
& \mathrm{~s}_{0} x^{\mu}=0 \quad \mathrm{~s}_{0} \theta^{\mu}=0 \quad \mathrm{~s}_{0} p_{\mu}=0 \quad \mathrm{~s}_{0} e=-\partial c \quad \mathrm{~s}_{0} \psi=\partial \gamma \\
& \mathrm{s}_{0} c=0 \quad \mathrm{~s}_{0} \gamma=0 .
\end{aligned}
$$

We see that the cohomology $E_{1}$ of the differential $\mathrm{s}_{0}$, is a graded polynomial ring in the following generators:

\begin{tabular}{|r|c|}
\hline gh & generators \\
\hline-1 & $\mathrm{E}^{+}=\int e^{+} d t, \Psi^{+}=\int \psi^{+} d t$ \\
0 & $\mathrm{X}^{\mu}=\int x^{\mu} d t, \Theta^{\mu}=\int \theta^{\mu} d t, \mathrm{P}_{\mu}=\int p_{\mu} d t$ \\
1 & $\mathrm{C}=\int c d t, \Gamma=\int \gamma d t$ \\
\hline
\end{tabular}

The differential $\mathrm{s}_{1}$ on $E_{1}$ is given by the formulas

$$
\mathrm{s}_{1} \mathrm{E}^{+}=-\frac{1}{2} \eta^{\mu \nu} \mathrm{P}_{\mu} \mathrm{P}_{\nu} \quad \mathrm{s}_{1} \Psi^{+}=-\mathrm{P}_{\mu} \Theta^{\mu}
$$


A cochain in $E_{1}$ has the general form

$$
z=\sum_{j>0}\left(\Psi^{+}\right)^{j-1} \mathrm{E}^{+} a_{j}+\sum_{j \geq 0}\left(\Psi^{+}\right)^{j} b_{j}
$$

where $a_{j}, b_{j} \in \mathcal{O}\left[\Theta^{\mu}, \mathrm{P}_{\mu}, \mathrm{C}, \Gamma\right]$. The differential of $z$ equals

$\mathrm{s}_{1} z=\sum_{j>0} j\left(\Psi^{+}\right)^{j-1} \mathrm{E}^{+}\left(\mathrm{P}_{\mu} \Theta^{\mu}\right) a_{j+1}-\sum_{j \geq 0}\left(\Psi^{+}\right)^{j}\left((j+1)\left(\mathrm{P}_{\mu} \Theta^{\mu}\right) b_{j+1}+\left(\frac{1}{2} \eta^{\mu \nu} \mathrm{P}_{\mu} \mathrm{P}_{\nu}\right) a_{j+1}\right)$.

The equations

$$
j\left(\mathrm{P}_{\mu} \Theta^{\mu}\right) b_{j}=-\left(\frac{1}{2} \eta^{\mu \nu} \mathrm{P}_{\mu} \mathrm{P}_{\nu}\right) a_{j}
$$

imply the vanishing of $\mathrm{s}_{1} z$. To see this, multiply both sides of the equation (7.1) by $\mathrm{P}_{\mu} \Theta^{\mu}$. The left-hand side vanishes: since $\eta^{\mu \nu} \mathrm{P}_{\mu} \mathrm{P}_{\nu}$ is not a zero-divisor, it follows that

$$
\left(\mathrm{P}_{\mu} \Theta^{\mu}\right) a_{j}=0 .
$$

Associate to $f \in \mathcal{O}[\mathrm{C}, \Gamma]$ the cocycles in $E_{1}$

$$
\mathbb{A}_{j}(f)=2 j\left(\Psi^{+}\right)^{j-1} \mathrm{E}^{+} f \Omega-\left(\Psi^{+}\right)^{j} f \iota(\mathrm{P}) \Omega, \quad \mathbb{B}_{j}(f)=\left(\Psi^{+}\right)^{j} f \Omega .
$$

Since the operation of multiplication by $\mathrm{P}_{\mu} \Theta^{\mu}$ may be interpreted as the differential of a Koszul complex, it has kernel spanned by elements of the form $\left(\mathrm{P}_{\mu} \Theta^{\mu}\right) u$, where $u \in \mathcal{O}\left[\Theta^{\mu}, \mathrm{P}_{\mu}, \mathrm{C}, \Gamma\right]$, and $f \Omega$, where $f \in \mathcal{O}[\mathrm{C}, \Gamma]$. Thus, we may decompose a solution $a_{j}$ of $(7.2)$ as a sum

$$
a_{j}=\left(\mathrm{P}_{\mu} \Theta^{\mu}\right) u_{j}+2 j f_{j} \Omega,
$$

where $u_{j} \in \mathcal{O}\left[\Theta^{\mu}, \mathrm{P}_{\mu}, \mathrm{C}, \Gamma\right]$ and $f_{j} \in \mathcal{O}[\mathrm{C}, \Gamma]$. This allows us to rewrite the cocycle $z$ as

$$
z=b_{0}+\sum_{j>0} \mathbb{A}_{j}\left(f_{j}\right)+\sum_{j>0}\left(\Psi^{+}\right)^{j}\left(b_{j}+f_{j} \iota(\mathrm{P}) \Omega+\left(\eta^{\mu \nu} \mathrm{P}_{\mu} \mathrm{P}_{\nu}\right) u_{j}\right)+\mathrm{s}_{1} \sum_{j>0} 2\left(\Psi^{+}\right)^{j} \mathrm{E}^{+} u_{j} .
$$

Applying (7.1), we see that

$$
\left(\mathrm{P}_{\mu} \Theta^{\mu}\right)\left(b_{j}+f_{j} \iota(\mathrm{P}) \Omega+\left(\eta^{\mu \nu} \mathrm{P}_{\mu} \mathrm{P}_{\nu}\right) u_{j}\right)=0
$$

Hence there is a decomposition

$$
b_{j}+f_{j} \iota(\mathrm{P}) \Omega+\left(\eta^{\mu \nu} \mathrm{P}_{\mu} \mathrm{P}_{\nu}\right) u_{j}=(j+1)\left(\mathrm{P}_{\mu} \Theta^{\mu}\right) v_{j}+g_{j} \Omega,
$$

where $g_{j} \in \mathcal{O}[\mathrm{C}, \Gamma]$. This allows us to rewrite the cocycle $z$ as

$$
z=b_{0}+\sum_{j>0}\left(\mathbb{A}_{j}\left(f_{j}\right)+\mathbb{B}_{j}\left(g_{j}\right)\right)+\mathrm{s}_{1} \sum_{j>0}\left(2\left(\Psi^{+}\right)^{j} \mathrm{E}^{+} u_{j}+\left(\Psi^{+}\right)^{j+1} v_{j}\right) .
$$

We conclude that a cohomology classes in $E_{2}=H^{*}\left(E_{1}, \mathrm{~s}_{1}\right)$ take the general form

$$
z=\left[b_{0}\right]+\sum_{j>0}\left(\left[\mathbb{A}_{j}\left(f_{j}\right)\right]+\left[\mathbb{B}_{j}\left(g_{j}\right)\right]\right)
$$


where $\left[b_{0}\right]$ is an element of the ring

$$
\mathcal{O}\left[\Theta^{\mu}, \mathrm{P}_{\mu}, \mathrm{C}, \Gamma\right] /\left(\mathrm{P}_{\mu} \Theta^{\mu}, \frac{1}{2} \eta^{\mu \nu} \mathrm{P}_{\mu} \mathrm{P}_{\nu}\right)
$$

The differential $\mathrm{s}_{2}$ on $E_{2}$ is induced by the graded differential taking the following values on the generators of $E_{1}$ :

$$
\begin{aligned}
\mathrm{s}_{2} \mathrm{E}^{+} & =0 & \mathrm{~s}_{2} \Psi^{+} & =2 \mathrm{E}^{+} \Gamma \\
\mathrm{s}_{2} \mathrm{X}^{\mu} & =-\eta^{\mu \nu} \mathrm{P}_{\nu} \mathrm{C}-\Theta^{\mu} \Gamma & \mathrm{s}_{2} \Theta^{\mu} & =-\eta^{\mu \nu} \mathrm{P}_{\nu} \Gamma \\
\mathrm{s}_{2} \mathrm{C} & =\Gamma^{2} & \mathrm{~s}_{2} \Gamma & =0 .
\end{aligned}
$$

We have

$$
\mathrm{s}_{2}\left(\mathbb{B}_{j}(f)\right)=\mathbb{A}_{j}(\Gamma f)-\mathrm{s}_{1}\left(\frac{\left(\Psi^{+}\right)^{j+1} C_{\iota}(\operatorname{grad} f) \Omega}{j+1}\right) .
$$

This establishes the formula $\mathrm{s}_{2}\left[\mathbb{B}_{j}(f)\right]=\left[\mathbb{A}_{j}(\Gamma f)\right]$ and $\mathrm{s}_{2}\left[\mathbb{A}_{j}(f)\right]=0$ in $E_{2}$. Thus, the subcomplex of $E_{2}$ spanned by

$$
\left\{\left[\mathbb{A}_{k}(f)\right],\left[\mathbb{B}_{k}(f)\right] \mid k>0, f \in \mathcal{O}[\mathrm{C}, \Gamma]\right\}
$$

contributes to $E_{3}$ a summand spanned by

$$
\left\{\left[\mathbb{A}_{k}(f)\right] \mid k>0, f \in \mathcal{O}[\mathrm{C}]\right\} .
$$

These elements of $E_{3}$ lift to the cocycles $\alpha_{k}(f)$ and $\zeta_{k}(f)$ in $\mathcal{A}$, showing that these classes in $E_{3}$ all survive to the last page $E_{\infty}$ of the spectral sequence.

It remains to calculate the contribution of the elements of the form $\left[b_{0}\right] \in E_{2}$ to $E_{3}$. There is a quasi-isomorphism between the complexes

$$
\left(\mathcal{O}\left[\Theta^{\mu}, \mathrm{P}_{\mu}, \mathrm{C}, \Gamma\right] /\left(\mathrm{P}_{\mu} \Theta^{\mu}, \eta^{\mu \nu} \mathrm{P}_{\mu} \mathrm{P}_{\nu}\right), \mathrm{s}_{2}\right)
$$

and $\mathcal{R}$, induced by sending the elements $C$ and $\Gamma^{2}$ to zero and $\Gamma$ to $-\Gamma$. Thus the subspace of $E_{2}$ consisting of elements of the form $\left[b_{0}\right]$ contributes a copy of the cohomology $H^{*}(\mathcal{R})$ of $\mathcal{R}$ to $E_{3}$. These classes lift to the summand of $H^{*}(\mathcal{A}, \mathrm{s})$ spanned by the classes

$$
\left\{\xi^{0}(u) \mid u \in H^{0}(\mathcal{R})\right\} \oplus\left\{\xi^{1}(v) \mid v \in H^{1}(\mathcal{R})\right\},
$$

completing the proof of the theorem.

We denote the transgressions of the cocycles $\alpha_{k}(f), \zeta_{k}(f), \xi^{0}(u)$ and $\xi^{1}(v)$ obtained by applying the vector field $\mathrm{g}$ by $\tilde{\alpha}_{k}(f), \tilde{\zeta}_{k}(f), \tilde{\xi}^{0}(u)$ and $\tilde{\xi}^{1}(v)$. The following corollary follows by applying the long-exact sequence (2.2). 


\section{Corollary.}

$$
H^{-k}(\mathcal{F}, \mathbf{s})=\left\{\begin{array}{rl}
\left\{\int\left(\alpha_{k}(f)+\zeta_{k}(g)+\tilde{\alpha}_{k}(\tilde{f})+\tilde{\zeta}_{k}(\tilde{g})\right) d t \mid f, g, \tilde{f}, \tilde{g} \in \mathcal{O}\right\} & k>1, \\
\left\{\int\left(\tilde{\xi}^{-1}(u)+\alpha_{1}(f)+\zeta_{1}(g)+\tilde{\alpha}_{1}(\tilde{f})+\tilde{\zeta}_{1}(\tilde{g})\right) d t \mid\right. & \\
\left.u \in H^{0}(\mathcal{R} / \mathbb{C}), f, g, \tilde{f} \in \mathcal{O}, \tilde{g} \in \mathcal{O} / \mathbb{C}\right\} & k=1, \\
\left\{\left(\xi^{0}(u)+\tilde{\xi}^{0}(v)+\alpha_{0}(f)+\zeta_{0}(g)\right) d t \mid\right. & k=0, \\
\left\{\int \xi^{1}(v) d t \mid v \in H^{1}(\mathcal{R})\right\} & k=-1, \\
0 & k<-1 .
\end{array}\right.
$$

In the remainder of this section, we calculate some examples of graded Lie brackets among the cohomology groups $H^{*}(\mathcal{F}, \mathrm{s})$. All of the brackets among classes in the image of $H^{*}(\mathcal{A}, \mathrm{s}) \rightarrow H^{*}(\mathcal{F}, \mathrm{s})$ vanish. As for brackets among the classes $\xi^{i}(u)$ and $\tilde{\xi}^{i}(u)$, we have $\left\{\xi^{i}(u), \xi^{j}(v)\right\}=0$, and

$$
\left\{\tilde{\xi}^{i}(u), \xi^{j}(v)\right\}=\xi^{i+j}(\{u, v\}), \quad\left\{\tilde{\xi}^{i}(u), \tilde{\xi}^{j}(v)\right\}=(-1)^{\mathrm{p}(u)} \tilde{\xi}^{i+j}(\{u, v\}),
$$

where

$$
\{u, v\}=(-1)^{\mathrm{p}(u)} \frac{\partial u}{\partial x^{\mu}} \frac{\partial v}{\partial p_{\mu}}-(-1)^{\mathrm{p}(u)} \frac{\partial u}{\partial p_{\mu}} \frac{\partial v}{\partial x^{\mu}}-\eta^{\mu \nu} \frac{\partial u}{\partial \theta^{\mu}} \frac{\partial v}{\partial \theta^{\nu}} .
$$

Here, we understand $\xi^{k}(u)$ and $\tilde{\xi}^{k}(u)$ to be 0 when $k>1$.

We may also calculate the bracket of $\tilde{\alpha}_{k}(f)$ with the classes $\alpha_{\ell}(g), \zeta_{\ell}(g), \xi^{i}(u)$, taking the calculation in the case $d=0$ as the model. We have

$$
\begin{aligned}
\mathrm{g}\left(A_{k}(f)\right)= & (k+1)\left(\psi^{+}\right)^{k} \gamma^{+} c f \Omega \gamma^{-1}-\left(\psi^{+}\right)^{k+1} e f \Omega \gamma^{-1}-\left(\psi^{+}\right)^{k+1}\left(p^{+} \cdot \operatorname{grad} f\right) c \Omega \gamma^{-1} \\
& +\left(\psi^{+}\right)^{k+1} c f \iota\left(\theta^{+}\right) \Omega \gamma^{-1}+(-1)^{d}\left(\psi^{+}\right)^{k+1} c f \Omega \psi \gamma^{-2}
\end{aligned}
$$

Using this formula, it is not hard to show that $\left\{\mathrm{g}\left(A_{k}(f)\right), \alpha_{\ell}(g)\right\}=\left\{\mathrm{g}\left(A_{k}(f)\right), \alpha_{\ell}(g)\right\}=0$, at least when $d>2$, and hence that $\left\{\tilde{\alpha}_{k}(f), \alpha_{\ell}(g)\right\}=\left\{\tilde{\alpha}_{k}(f), \zeta_{\ell}(g)\right\}=0$. When $d \leq 2$, the brackets $\left\{\mathrm{g}\left(A_{k}(f)\right), \alpha_{\ell}(g)\right\}$ and $\left\{\mathrm{g}\left(A_{k}(f)\right), \alpha_{\ell}(g)\right\}$ are coboundaries in the complex $\mathcal{A}$, leading to the same conclusion.

\section{Acknowledgments}

I am grateful to Chris Hull for introducing me to the first-order formalism of the spinning particle.

Igor Zavkhine informed me that an earlier version of this paper contained an incorrect calculation of $H^{-1}(\mathcal{F}, \mathrm{s})$. This revised version of the paper corrects this, and calculates the cohomology in positive degrees as well.

This research is partially supported by EPSRC Programme Grant EP/K034456/1 "New Geometric Structures from String Theory" and Collaboration Grant \#243025 of the Simons Foundation. 
Open Access. This article is distributed under the terms of the Creative Commons Attribution License (CC-BY 4.0), which permits any use, distribution and reproduction in any medium, provided the original author(s) and source are credited.

\section{References}

[1] G. Barnich, F. Brandt and M. Henneaux, Local BRST cohomology in gauge theories, Phys. Rept. 338 (2000) 439 [hep-th/0002245] [InSPIRE].

[2] L. Brink, S. Deser, B. Zumino, P. Di Vecchia and P.S. Howe, Local Supersymmetry for Spinning Particles, Phys. Lett. B 64 (1976) 435 [Erratum ibid. B 68 (1977) 488] [INSPIRE].

[3] K. Costello, Renormalization and effective field theory, American Mathematical Society, Providence RI U.S.A. (2011) [ISBN: 978-0-8218-5288-0, 978-1-4704-1397-2] [Math. Surveys Monogr. 170 (2011) 1].

[4] G. Felder, D. Kazhdan and T.M. Schlank, The classical master equation, in Perspectives in representation theory, American Mathematical Society, Providence RI U.S.A. (2014), Contemp. Math. 610 (2014) 79 [arXiv:1212.1631] [INSPIRE]. 